\title{
Universal quantum phase transition from superconducting to insulating-like states in pressurized $\mathrm{Bi} 2 \mathrm{Sr} 2 \mathrm{CaCu} 2 \mathrm{O} 8+\delta$ superconductors
}

Liling Sun ( $D$ llsun@iphy.ac.cn )

Institute of Physics, Chinese Academy of Sciences https://orcid.org/0000-0002-6653-7826

\section{Yazhou Zhou}

Institute of Physics, Chinese Academy of Sciences

Jing Guo

Institute of Physics, Chinese Academy of Sciences https://orcid.org/0000-0002-2164-5065

Shu Cai

Institute of Physics, Chinese Academy of Sciences

Jinyu Zhao

Institute of Physics, Chinese Academy of Sciences

Genda Gu

Brookhaven National Laboratory

Chengtian Lin

Max-Planck-Inst of Solid State Res

Hongtao Yan

Institute of Physics, Chinese Academy of Sciences

Cheng Huang

Institute of Physics, Chinese Academy of Sciences

Chongli Yang

Institute of Physics, Chinese Academy of Sciences

Sijin Long

Institute of Physics, Chinese Academy of Sciences

\section{Yu Gong}

Institute of Physics, Chinese Academy of Sciences

Yanchun Li

Institute of High Energy Physics https://orcid.org/0000-0003-4540-4546

Xiaodong Li

Institute of High Energy Physics https://orcid.org/0000-0002-2290-1198

Qi Wu

Institute of Physics, Chinese Academy of Sciences

Jiangping $\mathrm{Hu}$ 
Institute of Physics, Chinese Academy of Sciences

\section{Xingjiang Zhou}

National Lab for Superconductivity, Institute of Physics, Chinese Academy of Sciences https://orcid.org/0000-0002-5261-1386

\section{Tao Xiang}

Institute of Physics, Chinese Academy of Sciences, Beijing National Laboratory for Condensed Matter Physics

\section{Article}

Keywords: copper oxide, superconductionrs, Bi2Sr2CaCu2O8+ס, high-Tc superconductivity

Posted Date: June 9th, 2021

DOl: https://doi.org/10.21203/rs.3.rs-551119/v1

License: (c) (1) This work is licensed under a Creative Commons Attribution 4.0 International License. Read Full License

Version of Record: A version of this preprint was published at Nature Physics on February 17th, 2022. See the published version at https://doi.org/10.1038/s41567-022-01513-2. 


\title{
Universal quantum phase transition from superconducting to insulating-like states in pressurized $\mathrm{Bi}_{2} \mathrm{Sr}_{2} \mathrm{CaCu}_{2} \mathrm{O}_{8+\delta}$
}

\author{
superconductors
}

\author{
Yazhou Zhou ${ }^{1 *}$, Jing Guo ${ }^{1,6 *}$, Shu Cai ${ }^{1 *}$, Jinyu Zhao ${ }^{1,4}$, Genda $\mathrm{Gu}^{2}$, Chengtian Lin $^{3}$, Hongtao \\ Yan $^{1,4}$, Cheng Huang ${ }^{1,4}$, Chongli Yang ${ }^{1}$, Sijin Long ${ }^{1,4}$, Yu Gong ${ }^{5}$, Yanchun $\mathrm{Li}^{5}$, Xiaodong $\mathrm{Li}^{5}$, \\ Qi Wu ${ }^{1}$, Jiangping $\mathrm{Hu}^{1,4}$, Xingjiang Zhou ${ }^{1,4,6}$, Tao Xiang ${ }^{1,4}$ and Liling Sun $^{1,4,6} \dagger$ \\ ${ }^{I}$ Institute of Physics, National Laboratory for Condensed Matter Physics, Chinese Academy of Sciences, Beijing, \\ 100190, China \\ ${ }^{2}$ Condensed Matter Physics \& Materials Science Department, Brookhaven National Laboratory, NY, 11973-5000 \\ USA \\ ${ }^{3}$ Max-Planck-Institut für Festko"rperforschung, D-70569 Stuttgart, Germany \\ ${ }^{4}$ University of Chinese Academy of Sciences, Department of Physics, Beijing 100190, China \\ ${ }^{5}$ Institute of High Energy Physics, Chinese Academy of Science, Beijing 100049, China \\ ${ }^{6}$ Songshan Lake Materials Laboratory, Dongguan, Guangdong 523808, China
}

Copper oxide superconductors have been continually fascinating the communities of condensed matter physics and material sciences because they host the highest ambient-pressure superconducting transition temperature $\left(T_{c}\right)$ and mysterious physics ${ }^{1-3}$. Searching for the universal correlation between the superconducting state and its normal state or neighboring ground state is believed to be an effective way for finding clues to elucidate the underlying mechanism of the superconductivity. One of the common pictures for the copper oxide superconductors is that a well-behaved metallic phase will present after the superconductivity is entirely suppressed by chemical doping ${ }^{4-8}$ or application of the magnetic field ${ }^{9}$. Here, we report the first observation of universal quantum phase transition from superconducting state to insulating-like state under pressure in the under-, optimally- and over-doped $\mathrm{Bi}_{2} \mathrm{Sr}_{2} \mathrm{CaCu}_{2} \mathrm{O}_{8}+\delta \quad\left(\mathrm{Bi}_{2} 212\right)$ superconductors with two $\mathrm{CuO}_{2}$ planes in a unit cell. The same phenomenon has also been found in the $\mathrm{Bi}_{2} \mathrm{Sr}_{1.63} \mathrm{La}_{0.37} \mathrm{CuO}_{6+\delta}(\mathrm{Bi2201})$ superconductor with one $\mathrm{CuO}_{2}$ plane and the $\mathrm{Bi}_{2.1} \mathrm{Sr}_{1.9} \mathrm{Ca}_{2} \mathrm{Cu}_{3} \mathrm{O}_{10+\delta}\left(\mathrm{Bi}_{2223}\right)$ superconductor with three $\mathrm{CuO}_{2}$ planes in a unit cell. These results not only provide fresh information on the cuprate superconductors but also pose a new challenge for achieving unified 


\section{understandings on the mechanism of the high- $T_{c}$ superconductivity.}

Although a huge body of experimental investigations has been made for the copper oxide (cuprate) superconductors since they were discovered for more than thirty years 10,11 , the correlation between the superconducting state and its normal state or the neighboring ground state is widely debated ${ }^{2,6,12-14}$. By changing the chemical makeup of interleaved charge-reservoir layers, electrons can be added to or removed from the $\mathrm{CuO}_{2}$ planes, resulting in the suppression of the antiferromagnetic insulating state of the parent compound ${ }^{2}$. As the doping level reaches a critical one, superconductivity presents and its transition temperature $\left(T_{c}\right)$ grows to a maximum upon doping to an optimal one, then declines for higher doping, and finally vanishes at a maximum doping level ${ }^{2,5,7,9,15} . T_{c \text {-max }}$ are referred to as optimal-doped ones. It is important to recognize that once the superconducting state is completely suppressed by the chemical doping, the material undergoes a quantum phase transition from a superconducting state to a metallic state ${ }^{16-18}$. However, the detailed experimental studies on the breakdown of the quantum state in cuprates are still lacking, which may be crucial for understanding how the superconducting state melts into or emerges from its neighboring ground states.

Pressure is an alternative method of tuning superconductivity beyond the chemical doping or external magnetic field, and it can provide significant information on the evolution among superconductivity, electronic state, and crystal structure without changing the chemical composition. On the other hand, it can also provide valuable assistance in the search for the superconductors with higher values of $T_{c}$ at ambient pressure by the substitution of the smaller ions ${ }^{19}$. A notably successful application of 
this strategy leads to the discoveries of the important cuprate and Fe-based superconductors $11,20,21$. Therefore, high-pressure studies on superconductivity can benefit not only for searching new superconductors but also for deeper understandings on the correlation between the superconducting and its neighbor normal or ground states ${ }^{22-26}$. To reveal how the superconducting state or non-superconducting state develops, a central issue for understanding the high- $T_{c}$ superconductivity in cuprates, we performed a series of high-pressure investigations by employing our newly developed state-of-the-art technique, a combined in-situ high-pressure measurements of the resistance and alternating current $(a c)$ susceptibility for the same sample at the same pressure. The studied samples that have been investigated broadly by variety of methods ${ }^{26-31}$ are the under-doped (UD), optimally-doped (OP) and over-doped (OD) $\mathrm{Bi}_{2} \mathrm{Sr}_{2} \mathrm{CaCu}_{2} \mathrm{O}_{8+\delta}(\mathrm{Bi} 2212)$ superconductors with two $\mathrm{CuO}_{2}$ planes in a unit cell.

Figure 1 shows the results of temperature versus in-plane resistance for the UD sample with $T_{c}=74 \mathrm{~K}$ (Fig.1a), the OP sample with $T_{c}=91 \mathrm{~K}$ (Fig. 1b) and the OD sample with $T_{c}=82 \mathrm{~K}$ (Fig.1c) at different pressures. It is found that the onset $T_{c}$ of these samples exhibits the same high-pressure behavior: a slight increase initially and then a monotonous decrease upon elevating pressure until not detectable. Subsequently, an unexpected insulating-like state presents at a pressure $\left(P_{i}\right)$ of $34.3 \mathrm{GPa}$ for the UDdoped sample, 39.9 GPa for the OP sample and 42.2 GPa for the OD sample, respectively. And the insulating-like behavior becomes pronounced when the pressure is higher than the $P_{i}$, (Fig.1a-1c). It is a grand surprise because naively one expects that by applying pressure the bandwidth should increase and thereby the system should 
become more metallic, but instead it becomes insulating-like. We repeated the measurements on new samples and found the results are reproducible [see Supplementary Information].

To investigate whether the insulating behavior observed is due to pressure-induced cracks in the material, we performed the experiments for the over-doped Bi2212 sample, and found a reversible transition between the superconducting state and the insulatinglike state (see Supplementary Information). These results rule out the possibility that the transition from a superconducting state to an insulating-like state is caused by cracks.

The combined high-pressure measurements of $a c$ susceptibility and in-plane resistance were performed for the above three kinds of samples. As shown in Fig.2, the superconducting transitions of the samples detected by the $a c$ susceptibility can be clearly identified by the onset signal of the deviation from the almost constant background on the high-temperature side (see the blue plots) and the plunge of the resistance to zero (see the red plots). Upon compression to $34.3 \mathrm{GPa}$ for the UD sample, 39.9 GPa for the OP sample and 42.2 for the OD sample, the samples show an insulating-like behavior (see the red plots in Fig.2d, 2h and 2l) and no diamagnetic signal is captured by the $a c$ susceptibility measurements (see the blue plots in Fig.2d, $2 \mathrm{~h}$ and $2 \mathrm{l}$ ). These results manifest that the pressure induces a quantum phase transition from a superconducting state to an insulating-like state in all these superconductors.

We summarize the experimental results in the normalized pressure- $T_{c}$ phase diagram in the left panel of Fig.3, which is established on the basis of the pressure- $T_{c}$ phase diagrams of the UD, OP and OD Bi2212 samples (the right panels of Fig.3). The 
phase diagram for the three kinds of samples shows two distinct regions: the superconducting state (SC) and the insulating-like state (I), and demonstrates a universal quantum phase transition from the superconducting to the insulating-like states. It is seen that $T_{c}$ displays a slight increase initially within a small pressure range, and then a continuous decrease with elevating pressure until fully suppressed at a critical pressure $\left(P_{c}\right.$, the determination of $P_{c}$ can be found in the caption of Figure 3$)$, above which an insulating-like state emerges, as shown in the left panel of Fig.3 (the detail of the normalizing analysis can be found in the Supplementary information).

In order to know whether the quantum phase transition discovered in this study is a common phenomenon beyond the Bi2212 superconductors investigated, we conducted the same measurements on the $\mathrm{Bi}_{2} \mathrm{Sr}_{1.63} \mathrm{La}_{0.37} \mathrm{CuO}_{6+\delta} \quad(\mathrm{Bi} 2201)$ superconductor with one $\mathrm{CuO}_{2}$ plane and the $\mathrm{Bi}_{2} \mathrm{Sr}_{2} \mathrm{Ca}_{2} \mathrm{Cu}_{3} \mathrm{O}_{10+\delta}$ (Bi2223) superconductor with three $\mathrm{CuO}_{2}$ planes in a unit cell. The same phenomenon is also found in these superconductors (see Supplementary Information), indicating that the observed quantum phase transition is universal in these bismuth-bearing cuprate superconductors, regardless of the doping level and the number of $\mathrm{CuO}_{2}$ planes in a unit cell.

These results impact our knowledge about the cuprate superconductors that, after the superconducting state is destroyed, the sample should show a well-behaved metallic state because pressure generally increases the bandwidth. To clarify the possible origin that leads to the destruction of the superconducting state and the emergence of the insulating-like state under pressure, we carried out more experiments. 
First, we conducted the high-pressure synchrotron X-ray diffraction measurements at $50 \mathrm{~K}$ for the OD sample on beamline 4W2 at the Beijing Synchrotron Radiation Facility. Our results indicated that there is no structural phase transition in the range of pressure up to $43.1 \mathrm{GPa}$, except that the volume of the lattice is apparently compressed (see Supplementary Information). These results ruled out the possibility that the quantum phase transition from superconducting to insulating-like states connects with a pressure-induced structural phase transition.

Second, we measured the magnetoresistance $(M R)$ at $4 \mathrm{~K}$ for the compressed UD, OP and OD samples that host the insulating-like state. The magnetic field was applied perpendicular to the $a b$-plane of these samples. As shown in Fig.4a-c, the $M R$ of all the samples exhibits a positive effect, the in-plane resistance increases upon elevating magnetic field. Considering that the $M R$ is very weak $(\sim 1 \%)$ and the appearance of the insulating-like state is close to the superconducting-insulating transition, we presume that the origin of the positive $M R$ may be related to the superconducting fluctuation.

Third, we performed the high-pressure Hall coefficient $\left(R_{H}\right)$ measurements for the OD sample (Fig.4d) and find that $R_{H}(P)$ decreases remarkably with increasing pressure up to $\sim 18 \mathrm{GPa}$. Because the Hall resistance versus magnetic field displays a linear behavior in the pressure range investigated (Supplementary Information), a typical feature of the single band, the decrease of $R_{H}(P)$ below $18 \mathrm{GPa}$ ought to be associated with the enhancement of carrier density. However, $R_{H}$ remains almost unchanged for pressures ranging from $\sim 18 \mathrm{GPa}$ to $\sim 35 \mathrm{GPa}$ and then shows a slow increase from $\sim 35$ GPa to $48.3 \mathrm{GPa}$. No apparent change in $R_{H}(P)$ at $P_{c}=39.5 \mathrm{GPa}$ implies that the total 
density of charge carriers seems to remain in a steady state across the quantum criticality. The reproducible result is also obtained in the Bi2201 superconductor (see Supplemental Information).

It is noted that, unlike the usual insulator, the low-temperature resistance in the insulating-like state rises way too slowly to be exponential. We attempted to fit the low temperature resistance with exponential dependence and power law, but they fail [see Supplementary Information]. Slow rises of the kind have been found in the low temperature orthorhombic $\mathrm{La}_{2-\mathrm{x}} \mathrm{Sr}_{\mathrm{x}} \mathrm{CuO}_{4}, \mathrm{YiBa}_{2} \mathrm{Cu}_{3} \mathrm{O}_{7-\delta}$ cuprates and $\mathrm{La}_{1-\mathrm{x}} M_{\mathrm{x}} \mathrm{OBiS}$, which are perceived as quite mysterious ${ }^{32-35}$.

There is in fact no precedence anywhere else for such a transition from a superconducting state to an insulating-like state without a coincident structural phase transition. Therefore, some questions are raised naturally: why do the itinerant superconducting electrons become localized after the quantum phase transition, and what is the exotic pathway that results in the quantum phase transition? If considering the buckling of the $\mathrm{CuO}_{2}$ planes due to the asymmetric lattice structure around the planes and nonuniform deformation from the doped atoms, when pressure shrinks the lattice constant of the planes, the plane buckling should be enhanced, which may help to develop an exotic band structure with a reduction of the bandwidth ${ }^{36}$ and eventually result in the emergence of the insulating-like state. All the above are the attractive issues in searching for the new physics behind the pressure-induced quantum phase transition from a superconducting state to insulating-like state instead of to a metallic state, which deserves further investigation with other advanced experimental probes and 
sophisticated theoretical studies.

\section{Acknowledgements}

We thank Prof. Jan Zaanen and Prof. Yi Zhou for helpful discussions on this work. The work in China was supported by the National Key Research and Development Program of China (Grant No. 2017YFA0302900, 2016YFA0300300 and 2017YFA0303103), the NSF of China (Grants No. U2032214, 11888101 and 12004419) and the Strategic Priority Research Program (B) of the Chinese Academy of Sciences (Grant No. XDB25000000). We thank the support from the Users with Excellence Program of Hefei Science Center CAS (2020HSC-UE015). Part of the work is supported by the Synergic Extreme Condition User System. J. G. is grateful for support from the Youth Innovation Promotion Association of the CAS (2019008). The work in BNL was supported by the US Department of Energy, office of Basic Energy Sciences (contract No. desc0012704).

\section{Author contributions}

L.S., T.X. and Q.W. designed the study and supervised the project. G. G. grew the $\mathrm{Bi}_{2} \mathrm{Sr}_{2} \mathrm{CaCu}_{2} \mathrm{O}_{8+\delta}$ single crystals. H.T.Y and X.J.Z. grew the $\mathrm{Bi}_{2} \mathrm{Sr}_{1.63} \mathrm{La}_{0.37} \mathrm{CuO}_{6+\delta}$ single crystals. C.T. L. grew the $\mathrm{Bi}_{2} \mathrm{Sr}_{2} \mathrm{Ca}_{2} \mathrm{Cu}_{3} \mathrm{O}_{10+\delta}$ single crystals. Y.Z., J.G., S.C., and L.S. performed the high pressure resistance, $a c$ susceptibility and magnetroresistance measurements. J.G., Y.Z., Y.G., Y.C.L., X.D.L. and C.L.Y. performed the high pressure X-ray diffraction measurements. J.G, C.H. and S.J.L. performed the high 
pressure Hall measurements. L.S., T.X., Q.W., J.P.H. and Y.Z. wrote the manuscript in consultation with all authors.

\section{Author information}

The authors declare no competing financial interest. Correspondence and requests for materials should be addressed to L.S.(11sun@iphy.ac.cn). These authors with star (*) contributed equally to this work.

\section{References}

1 Chu, C. W., Deng, L. Z. \& Lv, B. Hole-doped cuprate high temperature superconductors. Physica C Supercond 514, 290-313 (2015).

2 Keimer, B., Kivelson, S. A., Norman, M. R., Uchida, S. \& Zaanen, J. From quantum matter to high-temperature superconductivity in copper oxides. Nature 518, 179-186 (2015).

3 Zaanen, J. Superconducting electrons go missing. Nature 536, 282-283 (2016).

4 Norman, M. R. \& Pépin, C. The electronic nature of high temperature cuprate superconductors. Rep. Prog. Phys. 66, 1547-1610 (2003).

5 Barišić, N. et al. Universal sheet resistance and revised phase diagram of the cuprate high-temperature superconductors. Proc. Natl. Acad. Sci. U.S.A. 110, $12235-12240$ (2013).

6 Božović, I., He, X., Wu, J. \& Bollinger, A. T. Dependence of the critical temperature in overdoped copper oxides on superfluid density. Nature 536, 309- 
311 (2016).

7 Greene, R. L., Mandal, P. R., Poniatowski, N. R. \& Sarkar, T. The Strange Metal State of the Electron-Doped Cuprates. Annu. Rev. Condens. Matter Phys. 11, 213-229 (2020).

8 Sacépé, B., Feigel'man, M. \& Klapwijk, T. M. Quantum breakdown of superconductivity in low-dimensional materials. Nat. Phys. 16, 734-746 (2020).

9 Proust, C. \& Taillefer, L. The Remarkable Underlying Ground States of Cuprate Superconductors. Annu. Rev. Condens. Matter Phys. 10, 409-429 (2019).

10 Bednorz, J. G. \& Müller, K. A. Possible high $T_{c}$ superconductivity in the Ba-LaCu-O system. Z Phys B Con Mat 64, 189-193 (1986).

$11 \mathrm{Wu}, \mathrm{M}$. K. et al. Superconductivity at $93 \mathrm{~K}$ in a new mixed-phase $\mathrm{Y}-\mathrm{Ba}-\mathrm{Cu}-\mathrm{O}$ compound system at ambient pressure. Phys. Rev. Lett. 58, 908-910 (1987).

12 Zaanen, J. et al. Towards a complete theory of high $T_{C}$. Nat. Phys. 2, 138-143 (2006).

13 Lee, P. A., Nagaosa, N. \& Wen, X.-G. Doping a Mott insulator: Physics of hightemperature superconductivity. Rev. Mod. Phys. 78, 17-85 (2006).

14 Zaanen, J. Planckian dissipation, minimal viscosity and the transport in cuprate strange metals. Scipost Phys 6, 061 (2019).

15 Nakamae, S. et al. Electronic ground state of heavily overdoped nonsuperconducting $\mathrm{La}_{2-\mathrm{x}} \mathrm{Sr}_{\mathrm{x}} \mathrm{CuO}_{4}$. Phys. Rev. B 68, 100502 (2003).

16 Hussey, N. E., Abdel-Jawad, M., Carrington, A., Mackenzie, A. P. \& Balicas, L. A coherent three-dimensional Fermi surface in a high-transition-temperature 
superconductor. Nature 425, 814-817 (2003).

17 Platé, M. et al. Fermi Surface and Quasiparticle Excitations of Overdoped $\mathrm{Tl}_{2} \mathrm{Ba}_{2} \mathrm{CuO}_{6+\delta}$. Phys. Rev. Lett. 95, 077001 (2005).

18 Vignolle, B. et al. Quantum oscillations in an overdoped high-Tc superconductor. Nature 455, 952-955 (2008).

19 Chu, C. W. et al. Evidence for superconductivity above $40 \mathrm{~K}$ in the La-Ba-CuO compound system. Phys. Rev. Lett. 58, 405-407 (1987).

20 Takahashi, H. et al. Superconductivity at $43 \mathrm{~K}$ in an iron-based layered compound $\mathrm{LaO}_{1-\mathrm{x}} \mathrm{F}_{\mathrm{x}} \mathrm{FeAs}$. Nature 453, 376-378 (2008).

21 Zhi-An, R. et al. Superconductivity at $55 \mathrm{~K}$ in Iron-Based F-Doped Layered Quaternary Compound $\mathrm{Sm}\left[\mathrm{O}_{1-\mathrm{x}} \mathrm{F}_{\mathrm{x}}\right] \mathrm{FeAs}$. Chinese Phys. Lett. 25, 2215-2216 $(2008)$

22 S. Schilling, J. in Frontiers of High Pressure Research II: Application of High Pressure to Low-Dimensional Novel Electronic Materials (eds Hans D. Hochheimer, Bogdan Kuchta, Peter K. Dorhout, \& Jeffery L. Yarger) 345-360 (Springer Netherlands, 2001).

23 Sun, L. et al. Re-emerging superconductivity at 48 kelvin in iron chalcogenides. Nature 483, 67 (2012).

24 Deng, L. et al. Higher superconducting transition temperature by breaking the universal pressure relation. Proc. Natl. Acad. Sci. U.S.A. 116, 2004-2008 (2019).

25 Guo, J. et al. Crossover from two-dimensional to three-dimensional superconducting states in bismuth-based cuprate superconductor. Nat. Phys. 16, 
295-300 (2020).

26 Parker, C. V. et al. Fluctuating stripes at the onset of the pseudogap in the high$T_{c}$ superconductor $\mathrm{Bi}_{2} \mathrm{Sr}_{2} \mathrm{CaCu}_{2} \mathrm{O}_{8+\mathrm{x}}$. Nature 468, 677-680 (2010).

27 Kondo, T. et al. Disentangling Cooper-pair formation above the transition temperature from the pseudogap state in the cuprates. Nat. Phys. 7, 21-25 (2011).

28 Zhao, H. et al. Charge-stripe crystal phase in an insulating cuprate. Nat. Mater. 18, 103-107 (2019).

29 Du, Z. et al. Imaging the energy gap modulations of the cuprate pair-densitywave state. Nature 580, 65-70 (2020).

30 Gao, Q. et al. Electronic Evolution from the Parent Mott Insulator to a Superconductor in Lightly Hole-Doped $\mathrm{Bi}_{2} \mathrm{Sr}_{2} \mathrm{CaCu}_{2} \mathrm{O}_{8+\delta}$. Chinese Phys. Lett. 37, 087402 (2020).

31 Adachi, T. et al. Magnetic-field effects on the charge-spin stripe order in La214 high- $T_{C}$ cuprates. J. Phys. Conf. Ser. 51, 259-262 (2006).

32 Muramatsu, T., Pham, D. \& Chu, C. W. A possible pressure-induced superconducting-semiconducting transition in nearly optimally doped single crystalline $\mathrm{YBa}_{2} \mathrm{Cu}_{3} \mathrm{O}_{7-\delta}$. Appl. Phys. Lett. 99, 052508 (2011).

33 Fang, Y., Yazici, D., Jeon, I. \& Maple, M. B. High-pressure effects on nonfluorinated $\mathbf{B i S} \mathbf{S}_{2}$-based superconductors $\mathbf{L a}_{1-\mathbf{x}} \boldsymbol{M}_{\mathbf{x}} \mathbf{O B i S _ { 2 }}(\boldsymbol{M}=\mathbf{T i}$ and $\mathrm{Th})$. Phys. Rev. B 96, 214505 (2017).

34 Moritomo, Y., Kuwahara, H. \& Tokura, Y. Bandwidth- and Doping-Dependent Pressure Effects on the Ferromagnetic Transition in Perovskite Manganites. $J$. 
Phys. Soc. Japan 66, 556-559 (1997).
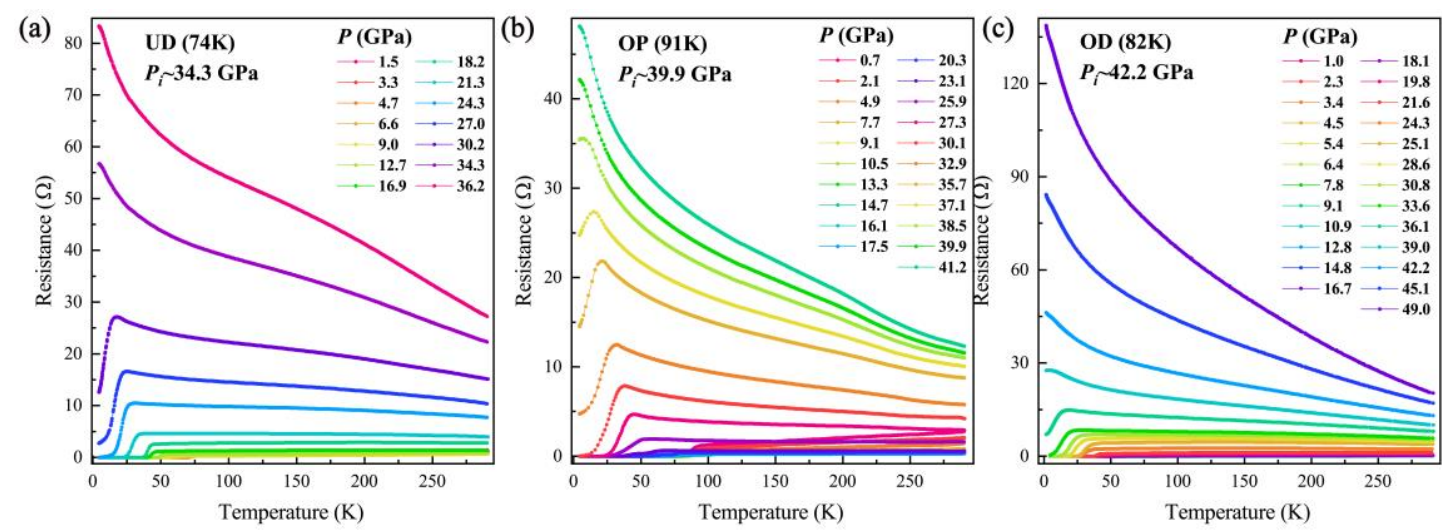

Figure 1 Temperature dependence of in-plane resistance for $\mathrm{Bi}_{2} \mathrm{Sr}_{2} \mathrm{CaCu}_{2} \mathrm{O}_{8}+\delta$

(Bi2212) at different pressures: (a) for the under-doped (UD) superconductor with superconducting transition temperature $\left(T_{c}\right)$ about $74 \mathrm{~K}$ in the pressure range of $1.5 \mathrm{GPa}$ - 36.2 GPa; (b) for the optimally-doped (OP) sample with $T_{c}$ about $91 \mathrm{~K}$ in the pressure range of $0.7 \mathrm{GPa}-41.2 \mathrm{GPa}$; (c) for the over-doped (OD) sample with $T_{c}$ about $82 \mathrm{~K}$ in the pressure range of $1.0 \mathrm{GPa}-49 \mathrm{GPa}$. The three kinds of samples display the same behavior of an insulating-like state above the pressure $\left(P_{i}\right)$. 

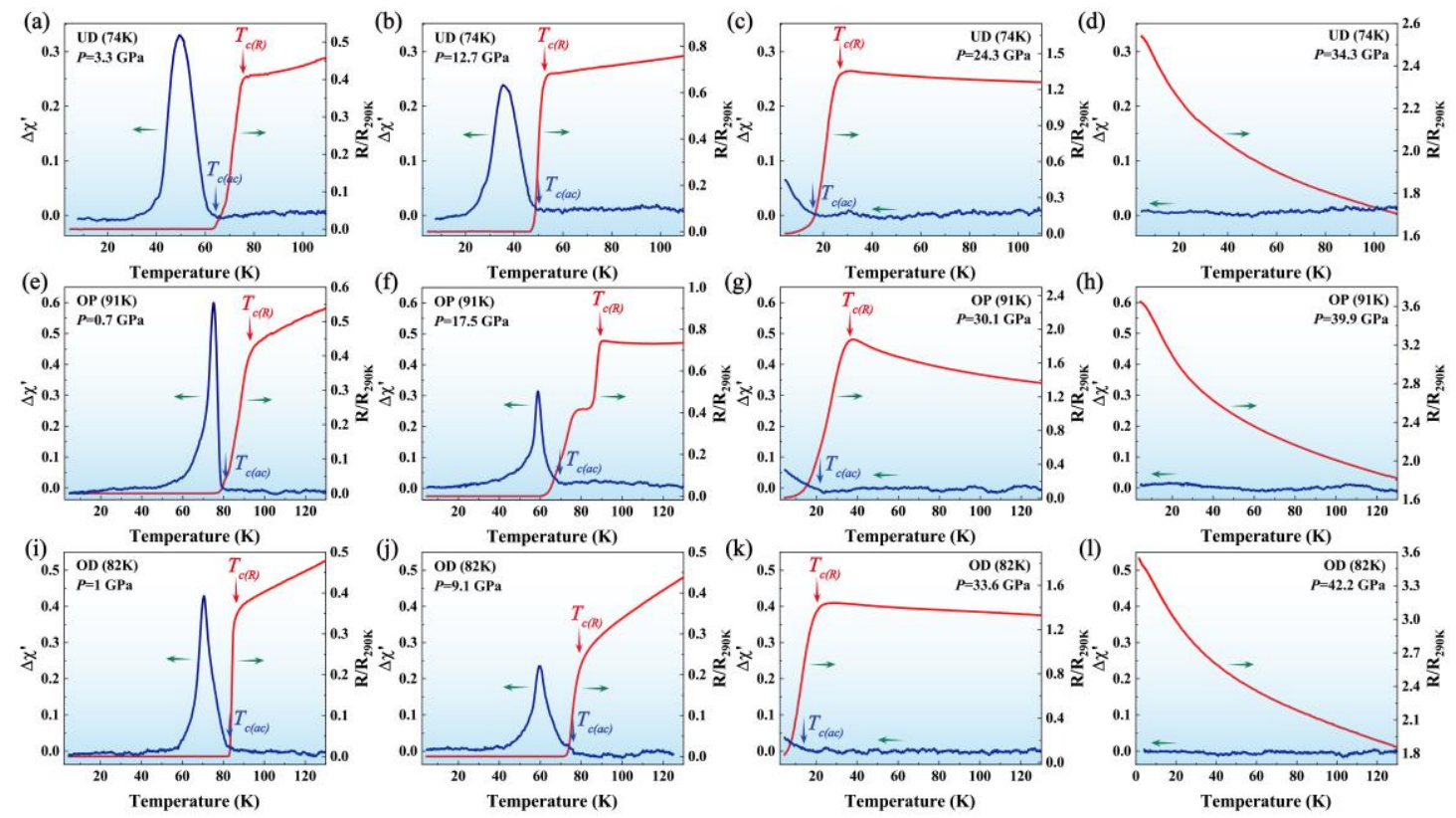

Figure 2. In-plane resistance $(R)$ and $a c$ susceptibility $\left(\Delta \chi^{\prime}\right)$ as a function of temperature ( $T$ ) for the $\mathrm{Bi}_{2} \mathrm{Sr}_{2} \mathrm{CaCu}_{2} \mathrm{O}_{8}+\delta$ superconductors at different pressures:

(a)-(d) for the under-doped (UD) superconductor; (e)-(h) for the optimally-doped (OP) superconductor; (i)-(l) for the over-doped (OD) superconductor. The blue lines in the figures are the data of $\Delta \chi^{\prime}(T)$, while the red lines are the data of $R(T)$. The red and blue arrows indicate the temperatures of the onset superconducting transition detected by resistance and $a c$ susceptibility measurements, respectively. 

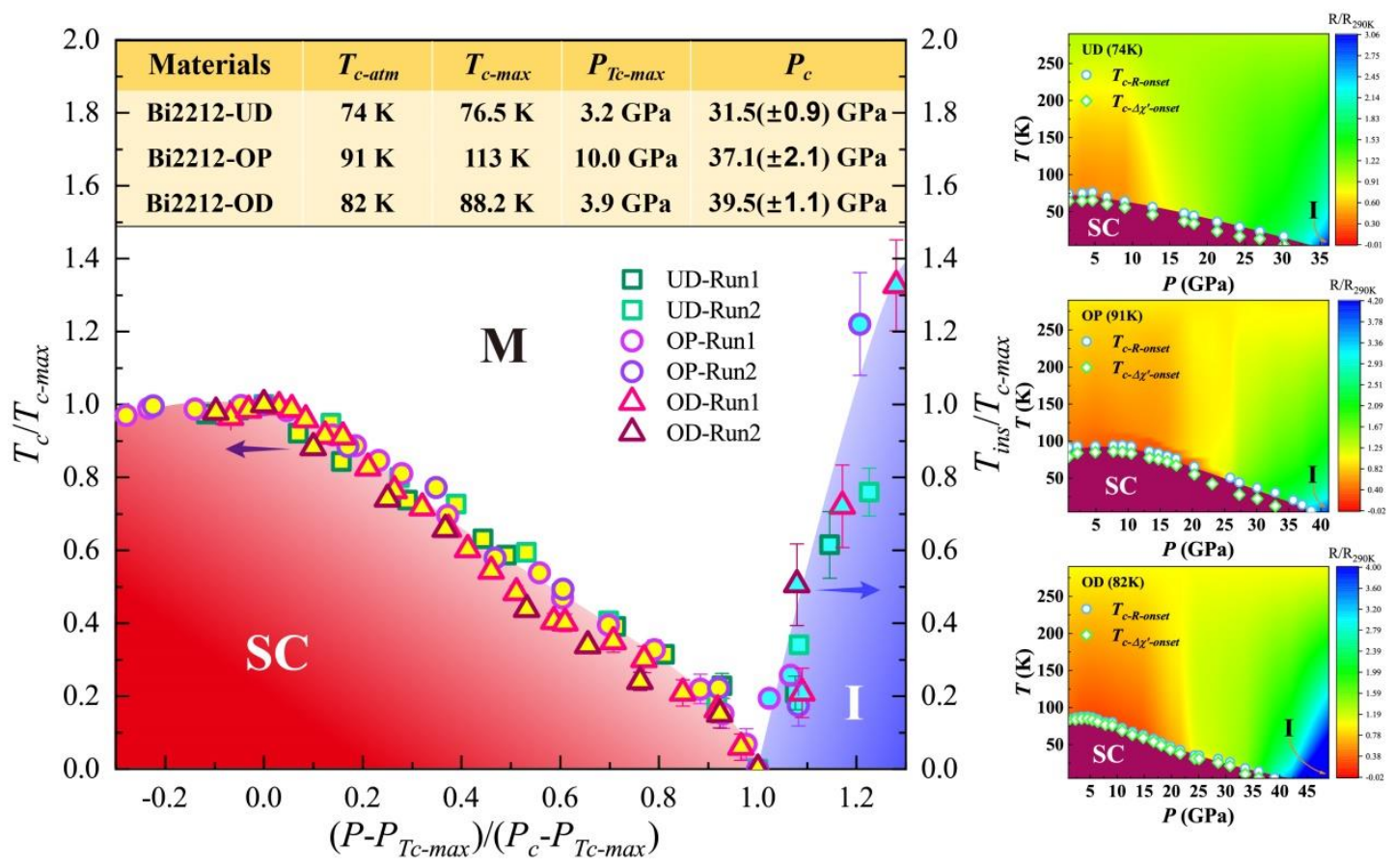

Figure 3 Pressure- $T_{c}$ phase diagrams for $\mathrm{Bi}_{2} \mathrm{Sr}_{2} \mathrm{CaCu}_{2} \mathrm{O}_{8+\delta}$ superconductors. The

Right panels are the phase diagrams established by the experimental results from the under-doped (UD), optimally-doped (OP) and over-doped (OD) samples, together with the mapping information of temperature and pressure dependent $R$ (shown in color scale). The left panel is a normalized phase diagram that is built on the basis of the experimental phase diagrams (the right panels). $P_{T c-\max }$ and $P_{c}$ stand for the critical pressures where $T_{c}$ reaches the maximum and the zero, respectively. In the normalizing analysis, we define the pressure as $P_{T c-\max }$ when $\left(P-P_{T c-\max }\right) /\left(P_{c}-P_{T c-\max }\right)=0$, and the pressure as $P_{c}$ when $\left(P-P_{T c-\max }\right) /\left(P_{c^{-}} P_{T c-\max }\right)=1$. The results of the normalizing analysis for $T_{c} / T_{c-\max }$ versus $\left(P-P_{T_{c-\max }}\right) /\left(P_{c-} P_{T c-\max }\right)$ and $T_{i n s} / T_{c-\max }$ versus $\left(P-P_{T c-\max }\right) /\left(P_{c-} P_{T c-\max }\right)$ show that the three kinds of samples display a universal quantum phase transition from the superconducting state to an insulating-like state. SC, M and I stand for superconducting state, metallic state and insulating-like state, respectively. The region of the M phase is determined by the critical value of $R / R_{290 K}$ where the quantum phase 
transition occurs. For example, when $R / R_{290 K}$ is greater than 3 , the over-doped sample is in the insulating-like state while, $R / R_{290 K}$ is less than 3 , the sample is in the metallic state (see right bottom panel). $T_{c-R \text {-onset }}$ and $T_{c-\Delta \chi^{\prime} \text {-onset }}$ denote the onset temperatures of the superconducting transition detected by the resistance and $a c$ susceptibility measurements, respectively. $T_{c-\max }$ and $T_{\text {ins }}$ are the maximum value of $T_{c}$ and the characteristic temperature of the insulating-like transition (the method of determining the $T_{\text {ins }}$ can be found in the Supplementary information), respectively. The $P_{c}$ value is determined by the average pressure of two experimental runs $\left[P_{c}=\left(P_{c-\text {-run } 1}^{\prime}+P_{c-\text { run } 2}^{\prime}\right) / 2\right]$, in which $P_{c}^{\prime}$ of each experimental run is determined by the highest experimental pressure where the superconducting transition can still be observed and the lowest experimental pressure where the insulating-like state appears. The error bar of $P_{c}$ is the difference between $P_{c \text {-run } 1}^{\prime}$ and $P_{c \text {-run } 2 \text {. }}^{\prime}$ 

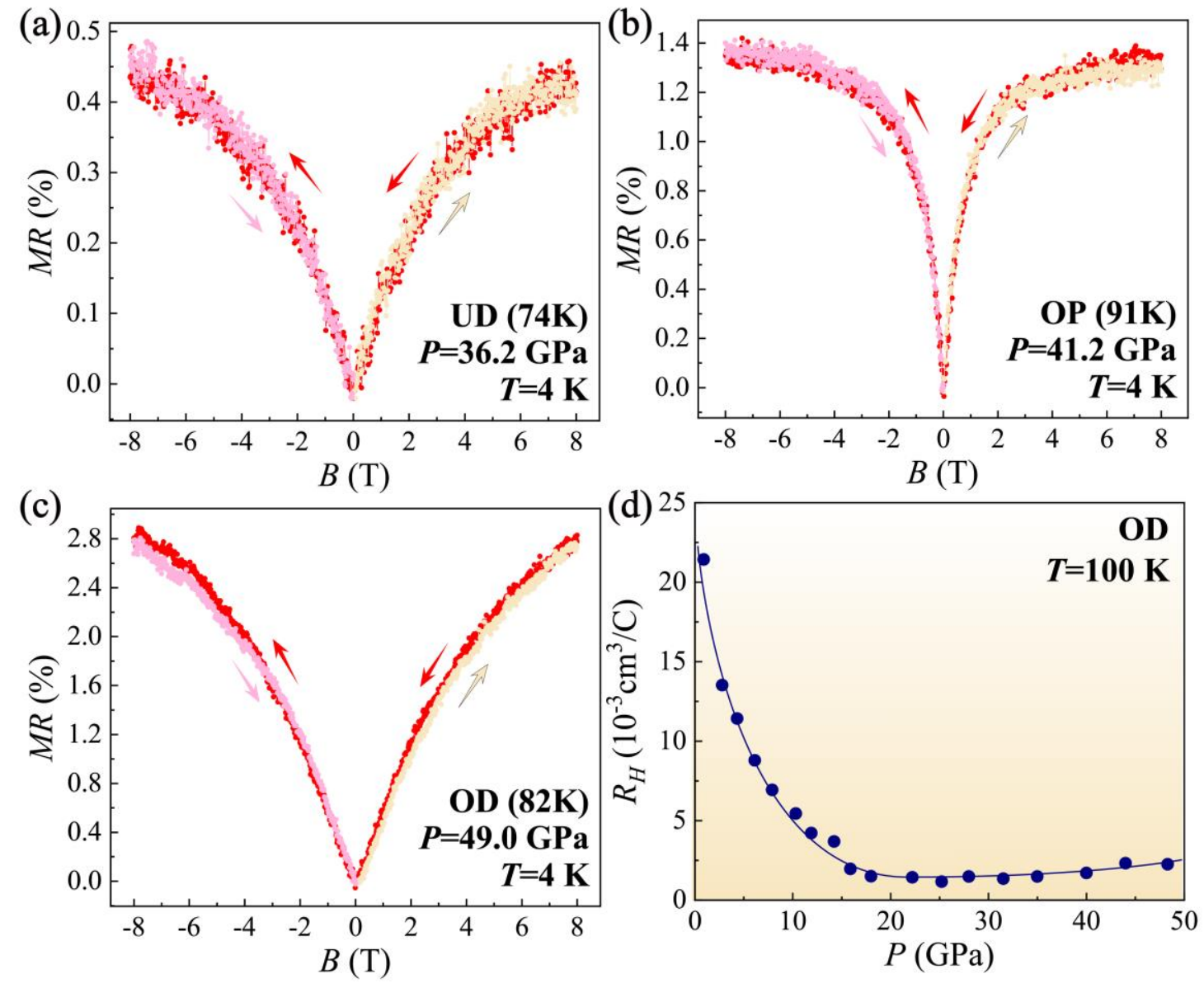

Figure 4 Magnetoresistance $(M R)$ as a function of magnetic field $(B)$ for the UD, $O P$ and $\mathrm{OD} \mathrm{Bi}_{2} \mathrm{Sr}_{2} \mathrm{CaCu}_{2} \mathrm{O}_{8+\delta}$ superconductors when they enter into an insulatinglike state, and Hall coefficient information of the OP sample under pressure. (a)(b) Plots of $M R$ versus $B$ for the UD, OP and OD samples measured at $4 \mathrm{~K}$ at $36.2 \mathrm{GPa}$, 41.2 $\mathrm{GPa}$ and $49 \mathrm{GPa}$, respectively. It is seen that all of them exhibit a positive magnetic effect. The red and beige arrows represent the directions of increasing and decreasing magnetic field. (d) Pressure dependence of Hall coefficient $\left(R_{H}\right)$ for the OD superconductor measured at $100 \mathrm{~K}$. 


\section{Supplementary Files}

This is a list of supplementary files associated with this preprint. Click to download.

- SIf.docx 